\title{
MicroRNA-199a-5p regulates FOXC2 to control human vascular smooth muscle cell phenotypic switch
}

\author{
YUSHI CAO ${ }^{1}$, ZHONGWEN CAO ${ }^{2}$, WEITIE WANG ${ }^{3}$, XIANGYU JIE ${ }^{2}$ and LEI $\mathrm{LI}^{2}$ \\ ${ }^{1}$ Department of Hepatobiliary Surgery, The First Hospital of Jilin University, Changchun, Jilin 130021; \\ ${ }^{2}$ Department of Vascular Surgery, Qianwei Hospital of Jilin Province, Changchun, Jilin 130012; \\ ${ }^{3}$ Department of Cardiovascular Surgery, The Second Hospital of Jilin University, Changchun, Jilin 130041, P.R. China
}

Received September 17, 2020; Accepted April 26, 2021

DOI: $10.3892 / \mathrm{mmr} .2021 .12266$

\begin{abstract}
Varicose veins are among the most common disorders of the vascular system; however, the pathogenesis of varicose veins remains unclear. The present study aimed to investigate the roles of microRNA (miR)-199a-5p in varicose veins and in the phenotypic transition of vascular smooth muscle cells (VSMCs). Bioinformatics analysis confirmed that miR-199a-5p had target sites on the forkhead box C2 (FOXC2) 3'-untranslated region. Reverse transcription-quantitative PCR (RT-qPCR) and western blotting were used to detect the expression levels of miR-199a-5p and FOXC2 in varicose vein and normal great saphenous vein tissues. Cell Counting Kit- 8 and Transwell migration assays were performed to validate the effects of miR-199a-5p on VSMCs. Contractile markers, such as smooth muscle $22 \alpha$, calponin, smooth muscle actin and myosin heavy chain 11 were used to detect phenotypic transition. RT-qPCR revealed that miR-199a-5p was downregulated in varicose veins compared with expression in normal great saphenous veins, whereas FOXC2 was upregulated in varicose veins. In addition, biomarkers of the VSMC contractile phenotype were downregulated in varicose veins. Overexpression of miR-199a-5p by mimics suppressed VSMC proliferation and migration, whereas depletion of miR-199a-5p enhanced VSMC proliferation and migration. Notably, the effects caused by miR-199a-5p could be reversed by FOXC2 overexpression. Dual luciferase reporter analysis confirmed that FOXC2 was a target of miR-199a-5p. In conclusion, miR-199a-5p may be a novel regulator of phenotypic switching in VSMCs by targeting FOXC2 during varicose vein formation.
\end{abstract}

Correspondence to: Dr Zhongwen Cao, Department of Vascular Surgery, Qianwei Hospital of Jilin Province, 1445 Qianjin Street, Changchun, Jilin 130012, P.R. China

E-mail:czwxwk@sina.com

Abbreviations: CCK-8, Cell Counting Kit-8; FOXC2, forkhead box $\mathrm{C} 2$; miRNA, microRNA; RT-qPCR, reverse transcriptionquantitative PCR; VSMCs, vascular smooth muscle cells

Key words: varicose veins, VSMCs, miRNA-199a-5p, FOXC2

\section{Introduction}

Lower varicose veins are a common disorder that mainly affect the great saphenous vein (1). Vascular smooth muscle cells (VSMCs) are the main cellular components of the normal vein wall, and phenotypic transition of VSMCs is a pathophysiological process that occurs in vascular diseases. Previous studies have reported that the transition of VSMCs from a contractile phenotype to a synthetic phenotype may lead to vascular remodeling and subsequently result in varicose veins $(2,3)$.

MicroRNAs (miRNAs/miRs) exhibit different expression levels in varicose veins compared with in normal veins (4). Notably, miR-199a-5p has been reported to participate in several pathological processes, such as osteoclast differentiation (5), spinal cord injury (6) and tumor proliferation (7). In addition, overexpression of miR-199a-5p has been shown to promote pulmonary artery hypertension by downregulating Smad3 in a previous study (8). Moreover, VSMCs transfected with miR-199a-5p mimics could inhibit nitric oxide levels and increase $\mathrm{Ca}^{2+}$ levels (8). Huang et al (9) transfected human airway smooth muscle cells with miR-199a-5p mimics and revealed that miR-199a-5p upregulation may contribute to neutrophilic asthma pathogenesis by modulating the inflammatory process. However, the role of miR-199a-5p and VSMC phenotypic transition in varicose veins remains unclear.

miR-199a-5p can suppress mRNA expression by directly targeting the 3 '-untranslated region (3'-UTR) $(5,6,8,9)$. Notably, forkhead box C2 (FOXC2) is a predicted target of miR-199a-5p (10). FOXC2 is a key transcription factor that participates in regulating adipose cell metabolism, and serves an important role in blood vessel and lymphatic vessel development (11). A recent report revealed that FOXC2 may be associated with venous valve dysfunction (12). In addition, FOXC2 has been reported to be upregulated in venous cells and to promote high expression of Notch pathway-related proteins (Dl14 and Hey2) (13). Notably, the Notch pathway has an essential role in the development of vascular networks (14). These findings indicated that miR-199a-5p/FOXC2 may be involved in varicose vein pathogenesis.

The present study aimed to investigate the roles of miR-199a-5p in varicose veins and in the phenotypic transition of VSMCs. Bioinformatics analysis was performed to identify 
the potential roles of miR-199a-5p/FOXC2 in varicose vein pathogenesis. The miR-199a-5p/FOXC2 axis may provide a novel mechanistic insight into the pathogenesis of varicose veins and may serve as a promising diagnostic biomarker and therapeutic target for the treatment of varicose veins.

\section{Materials and methods}

Tissue collection. The present study was conducted in accordance with the Declaration of Helsinki and was approved by the Ethics Committee of Qianwei Hospital of Jilin Province (Changchun, China; approval no. QW202000224). The varicose vein tissues were obtained from patients with varicose veins $(n=10$; average age, $63.2 \pm 8.9$ years; six patients were female; four had been diagnosed with hypertension; and one had been diagnosed with diabetes mellitus). Normal great saphenous vein tissues were obtained from individuals undergoing coronary artery bypass grafting surgery $(n=10$; average age, 63.6 \pm 8.7 years; five patients were female; five had been diagnosed with hypertension; and two had been diagnosed with diabetes mellitus). The inclusion and exclusion criteria of varicose vein was based on the criteria from the Updated 2020 Clinical, Etiologic, Anatomic and Pathophysiologic (CEAP) classification guidelines (15). All patients agreed to the use of their samples in scientific research and provided written informed consent.

Bioinformatics analysis. TargetScan (version 7.1; www. targetscan.org) online tool (10) was used to predict the relevant miRNAs that had binding sites with FOXC2 (Table SI). Conserved miRNAs were used for further confirmation. miR-199a was selected as the target miRNA as it has been more widely studied than miR-199b.

VSMC transfection. miR-199a-5p mimics and pcDNA3.1FOXC2 vector were used to induce the overexpression of miR-199a-5p and FOXC2, respectively. miR-199a-5p inhibitor and small interfering (si)RNA against FOXC2 (si-FOXC2) were used to knock down miR-199a-5p and FOXC2, respectively. Negative control (NC) mimics, NC inhibitor, pCDNA3.1 NC (empty plasmid) and NC siRNA (siNC) were used as the control groups. All mimics, inhibitors, plasmids and siRNAs were designed and synthesized by Shanghai GenePharma Co., Ltd. miR-199a-5p mimics and inhibitor were tagged with FAM. Lipofectamine $^{\circledR} 2000$ (Invitrogen; Thermo Fisher Scientific, Inc.) was used to transfect VSMCs (70-80\% confluence) with $250 \mathrm{pmol}(0.5 \mu \mathrm{g}) \mathrm{mimic}$ or inhibitor for $\sim 5 \mathrm{~h}$ at $37^{\circ} \mathrm{C}$. Total RNA and protein was harvested $48 \mathrm{~h}$ post-transfection. All experiments were conducted $48 \mathrm{~h}$ post-transfection. Sequences were as follows: miR-199a-5p mimics forward, 5'-CCCAGU GUUCAGACUACCUGUUC-3' and reverse, 5'-ACAGGU AGUCUGAACACUGGGUU-3'; NC mimics forward, 5'-UUC UCCGAACGUGUCACGUTT-3' and reverse, 5'-ACGUGA CACGUUCGGAGAATT-3'; miR-199a-5p inhibitor, 5'-GAA CAGGUAGUCUGAACACUGGG-3'; NC inhibitor, 5'-CAG UACUUUUGUGUAGUACAA-3'; si-FOXC2 forward, 5'-CUA CCUGAGCGAGCAGGUAGTT-3' and reverse, 5'-AUUCUG CUCGCUCAGGUAGTT-3'; and NC siRNA forward, 5'-UUC UCCGAACGUGUCACGUTT-3' and reverse, 5'-ACGUGA CACGUUCGGAGAATT-3'.
$R T-q P C R$. RNA was extracted from transfected or normal VSMCs and tissues using TRIzol ${ }^{\circledR}$ reagent (Invitrogen; Thermo Fisher Scientific, Inc.). A NanoDrop ND-1000 (NanoDrop; Thermo Fisher Scientific, Inc.) was used to detect the integrity and concentration of the RNA samples. Total RNA was reverse transcribed to cDNA using PrimeScript RT reagent kit with gDNA Eraser (Takara Bio, Inc.) according to the manufacturer's instructions. qPCR was performed using FastStart Universal SYBR Green Master (ROX) (Roche Diagnostics) and an Applied Biosystems 7500 Fast Real-Time PCR system (Applied Biosystems; Thermo Fisher Scientific, Inc.) to confirm the relative expression levels of miR-199a-5p and FOXC2. qPCR thermocycling conditions were as follows: Initial denaturation at $95^{\circ} \mathrm{C}$ for $5 \mathrm{~min}$; followed by 40 cycles at $95^{\circ} \mathrm{C}$ for $10 \mathrm{sec}, 55^{\circ} \mathrm{C}$ for $20 \mathrm{sec}$ and $72^{\circ} \mathrm{C}$ for $20 \mathrm{sec}$, and a final extension step at $72^{\circ} \mathrm{C}$ for $2 \mathrm{~min}$. The relative expression levels of genes were calculated using the $2^{-\Delta \Delta \mathrm{Cq}}$ method (16) after normalization to GAPDH (used for mRNA normalization) or U6 (used for miRNA normalization). The primer sequences were as follows: FOXC2 forward, 5'-CGGCCCAGCAGC AAACTTTCC-3' and reverse, 5'-AGAGGCGGCGTGGAT CTGTAG-3'; GAPDH forward, 5'-CGGACCAATACGACC AAATCCG' and reverse, 5'-AGCCACATCGCTCAGACA CC-3'; miR-199a-5p forward, 5'-CCGGGATCCGCAAAC TCAGCTTTAC-3' and reverse, 5'-CGGAATTCGTGGCGA CCGTGATACC-3'; U6 forward, 5'-GCGCGTCGTGAAGCG TTC-3' and reverse, 5'-GTGCAGGGTCCGAGGT-3'; SM22 $\alpha$ forward, 5'-AACAGCCTGTACCCTGATGG-3' and reverse, 5'-CGGTAGTGCCCATCATTCTT-3'; Calponin forward, 5'-AGCTAAGAGAAGGGCGGAAC-3' and reverse, 5'-CAT CTGCAGGCTGACATTGA-3'; SMA forward, 5'-GCGTGG CTATTCCTTCGTTA-3' and reverse, 5'-ATGAAGGATGGC TGGAACAG-3'; and Myosin Heavy Chain 11 (MYH11) forward, 5'-TGGAACTTCATCGACTTTGGG-3' and reverse, 5'-ACAGCTTCTCCACGAAAGAC-3'.

Cell culture. The human VSMC cell line was purchased from the Shanghai Institute of Biochemistry and Cell Biology and cultured in DMEM (Gibco; Thermo Fisher Scientific, Inc.) supplemented with $10 \%$ fetal bovine serum (Gibco; Thermo Fisher Scientific, Inc.) at $37^{\circ} \mathrm{C}$ in a humidified incubator containing $5 \% \mathrm{CO}_{2}$. The medium was replaced every 3 days. All cells used in the present study were between passages 5 and 7 . VSMCs were serum starved $(0.5 \%$ fetal bovine serum) for $24 \mathrm{~h}$ and stimulated using platelet-derived growth factor (PDGF)-BB (20 ng/ml; BioVision, Inc.) at $37^{\circ} \mathrm{C}$ for $24 \mathrm{~h}$ prior to experimentation.

Western blot analysis. Proteins were extracted from cells and tissues using RIPA buffer (Beijing Solarbio Science \& Technology Co., Ltd.) and were quantified using a BCA assay (Thermo Fisher Scientific, Inc.). Total cell proteins (30 $\mu \mathrm{g} / \mathrm{lane})$ were separated by SDS-PAGE on $10 \%$ gels (cat. no. P1200; Beijing Solarbio Science \& Technology Co., Ltd.) and transferred to nitrocellulose membranes $(0.45 \mu \mathrm{m}$; EMD Millipore). Blots were blocked with $5 \%$ nonfat milk with $0.1 \%$ Tween-20 (Sigma-Aldrich; Merck KGaA) for $2 \mathrm{~h}$ at $37^{\circ} \mathrm{C}$. Subsequently, membranes were incubated overnight at $4^{\circ} \mathrm{C}$ with the following primary antibodies: FOXC2 (1:500; cat. no. ab245510; Abcam), smooth muscle $22 \alpha$ (SM22 $\alpha$; 1:500; cat. no. 10493-1-AP; 
ProteinTech Group, Inc.), smooth muscle actin (SMA; 1:800; cat.no.55135-1-AP; ProteinTech Group, Inc.), MYH11 (1:1,000; cat. no. 21404-1-AP; ProteinTech Group, Inc.), calponin (1:1,000; cat. no. bs-0095R; BIOSS), proliferating cell nuclear antigen (PCNA; 1:3,000; cat. no. ab92552; Abcam) and $\beta$-actin (1:5,000; cat. no. bs-0061R; BIOSS), followed by incubation with secondary antibodies (1:5,000; cat. no. bs-0296G-HRP; BIOSS) at room temperature for $2.5 \mathrm{~h}$. Protein bands were visualized with an ECL western blotting kit (Cell Signaling Technology, Inc.) using cSeries Imager (Azure Biosystems, Inc.). Protein expression levels were semi-quantified using ImageJ software (version 4.62; National Institutes of Health).

Dual luciferase reporter assay. 293T cells were purchased from the Shanghai Institute of Biochemistry and Cell Biology. The wild-type (WT) or mutant (MUT) FOXC2 3'-UTRs were cloned into the pGL6-miR vector (Beyotime Institute of Biotechnology). FOXC2 mutation was induced using the Quick-Change Site-Directed Mutagenesis kit (Agilent Technologies, Inc.). Subsequently, $2 \times 10^{4} 293 \mathrm{~T}$ cells per well were seeded into 6-well plates for $24 \mathrm{~h}$ at $37^{\circ} \mathrm{C}$ before transfection. A Dual Luciferase Reporter Gene Assay kit (Promega Corporation) was used to assess luciferase activity after 293T cells were co-transfected with either pGL6-FOXC2-WT $(0.5 \mu \mathrm{g})$ or pGL6-FOXC2-MUT $(0.5 \mu \mathrm{g})$ and either miR-199a-5p mimics $(0.5 \mu \mathrm{g})$ or mimics NC $(0.5 \mu \mathrm{g})$ using Lipofectamine 2000. A total of $24 \mathrm{~h}$ post-transfection, luciferase activity was measured at $560 \mathrm{~nm}$. Luciferase activity was normalized to Renilla luciferase.

Cell proliferation assay. The proliferation of VSMCs transfected with miR-199a-5p mimics or inhibitor was determined using the Cell Counting Kit-8 (CCK-8) assay (TransGen Biotech Co., Ltd.) according to the manufacturer's instructions. Cells $\left(3 \times 10^{4}\right.$ per well) transfected with miR-199a-5p mimics or inhibitor were seeded into a 96-well plate for 24 , 48 and 72 h. Subsequently, $10 \mu$ l CCK-8 Solution was added to the medium and incubated for $4 \mathrm{~h}$ at $37^{\circ} \mathrm{C}$. The absorbance was detected at $450 \mathrm{~nm}$ using an Epoch microplate (Bio-Tek Instruments, Inc.).

Transwell migration assay. Cell migration was assessed by performing a Transwell migration assay using Transwell chambers with $8-\mu \mathrm{m}$ filter inserts (24-well inserts). Transfected cells $\left(5 \times 10^{4}\right)$ cultured in serum-free medium were seeded into the upper chamber of Transwell inserts, whereas $500 \mu 1$ DMEM supplemented with $20 \%$ FBS was added to the lower chamber. After $36 \mathrm{~h}$ at $37^{\circ} \mathrm{C}$, a cotton swab was used to remove the cells remaining in the upper chamber. Cells that had migrated to the lower chamber were fixed with $100 \%$ methanol at $37^{\circ} \mathrm{C}$ and stained with $0.1 \%$ crystal violet. The number of migrated cells was then counted under a laser scanning confocal microscope (magnification, x40; Leica Microsystems $\mathrm{GmbH}$ ).

Wound healing assay. Cells $\left(2.5 \times 10^{5}\right.$ cells/well) were seeded into a 6-well plate and transfected with miR-199a-5p mimics or inhibitor for $48 \mathrm{~h}$. Cells underwent serum starvation in medium containing $0.5 \%$ FBS for $48 \mathrm{~h}$, after which, a linear wound was created in the center of the cell layer using a 200- $\mu 1$ pipette tip. Cells were then incubated in serum-free medium for $48 \mathrm{~h}$. The floating cells were removed by washing the cells with PBS. The distance of cell migration was determined by the mean value of the width of the gap between the top, middle and bottom of the wound. The wounds were observed in five fields of view using a light microscope (magnification, x100).

Statistical analysis. All statistical analyses were performed using SPSS 20.0 (IBM Corp.). Data are presented as the mean \pm standard deviation. The experiments were repeated at least three times. Comparisons among groups were analyzed using one-way ANOVA followed by Bonferroni's post hoc test, or a paired Student's t-test for comparison of differences between two groups. $\mathrm{P}<0.05$ was considered to indicate a statistically significant difference.

\section{Results}

miR-199a-5p regulates VSMC phenotypic transition. RT-qPCR analysis revealed that the mRNA expression levels of phenotypic transition biomarkers, such as SM22 $\alpha$, calponin, SMA and MYH11, were decreased in varicose vein tissues compared with those in normal great saphenous vein tissues (Fig. 1A). Western blot analysis revealed that the protein expression levels of SM22 $\alpha$, calponin, SMA and MYH11 were also decreased in varicose vein tissues compared with those in normal great saphenous vein tissues (Fig. 1B). RT-qPCR analysis revealed that miR-199a-5p was downregulated in varicose vein tissues, whereas FOXC2 was upregulated in varicose vein tissues compared with that in normal great saphenous vein tissues (Fig. 1C). In addition, western blot analysis confirmed that the protein expression levels of FOXC2 were increased in varicose vein tissues compared with those in normal great saphenous vein tissues (Fig. 1B). These results indicated that miR-199a-5p may be involved in VSMC proliferation during varicose vein pathogenesis and could regulate VSMC phenotypic transition. The transition of VSMCs from a contractile phenotype to a synthetic phenotype was inferred from the expression levels of phenotypic transition-related biomarkers.

Downregulation of miR-199a-5p in proliferative VSMCs. To verify the finding that miR-199a-5p may participate in the regulation of VSMC proliferation, the expression levels of miR-199a-5p and FOXC2 were detected in proliferating VSMCs at 12, 24, 36, 48 and $60 \mathrm{~h}$ after stimulation with PDGF-BB (20 ng/ml) (17). miR-199a-5p (Fig. 2A) and SM22 $\alpha$ mRNA (Fig. 2B) expression levels were downregulated during VSMC proliferation, whereas FOXC2 mRNA expression was upregulated (Fig. 2C). In addition, western blot analysis confirmed that SM22 $\alpha$ was downregulated, but FOXC2 was upregulated in a time-dependent manner (Fig. 2D). These results indicated that miR-199a-5p was downregulated during VSMC proliferation in a time-dependent manner.

miR-199a-5p regulates the expression of phenotypic transition biomarkers involved in VSMC differentiation. VSMCs were transfected with miR-199a-5p mimics or miR-199a-5p inhibitor. Confirmation of transfection of VSMCs with the FAM-labeled miR-199a-5p mimics and miR-199a-5p inhibitor is shown in Fig. S1A and B, respectively. The transfection efficiency of miR-199a-5p mimics and inhibitor was assessed by RT-qPCR; 
A

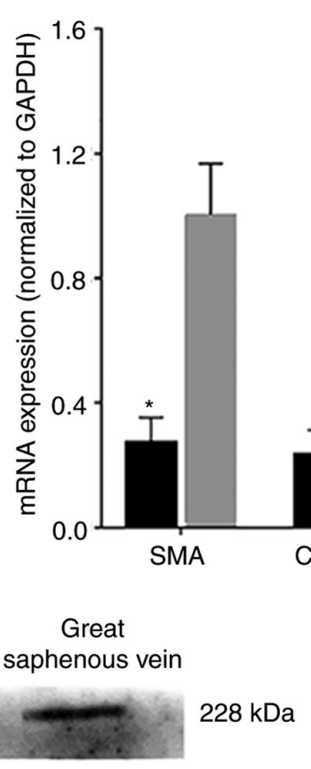

B

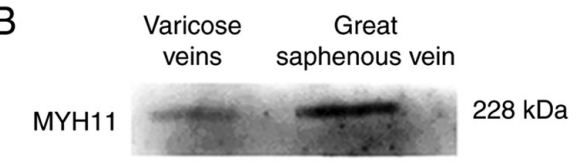

SMA

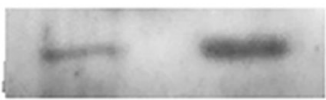

$42 \mathrm{kDa}$

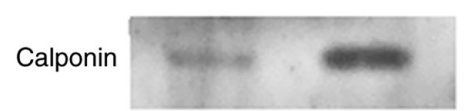

$33 \mathrm{kDa}$

$\operatorname{SM} 22 \alpha$

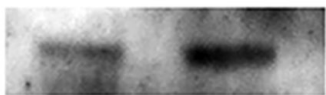

$22 \mathrm{kDa}$

FOXC2

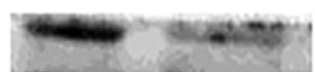

$56 \mathrm{kDa}$

$\beta$-actin

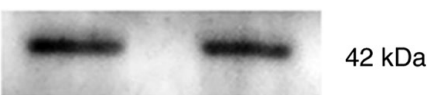

Varicose veins

Great saphenous vein
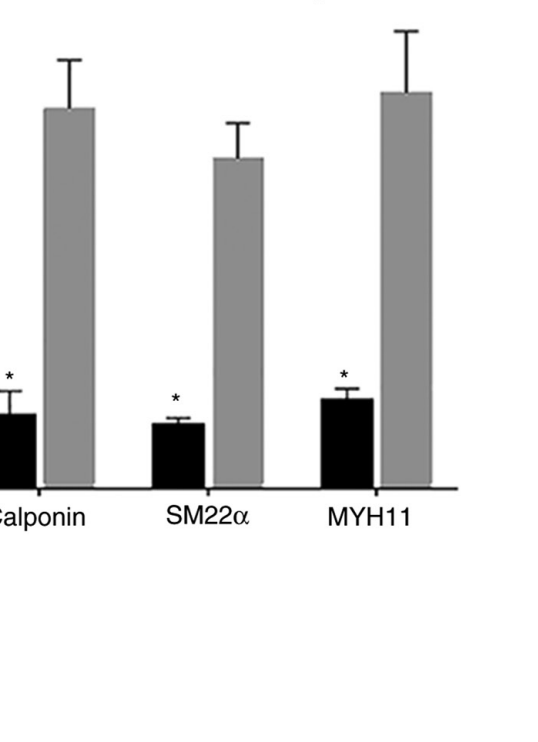

Varicose veins

Great saphenous vein
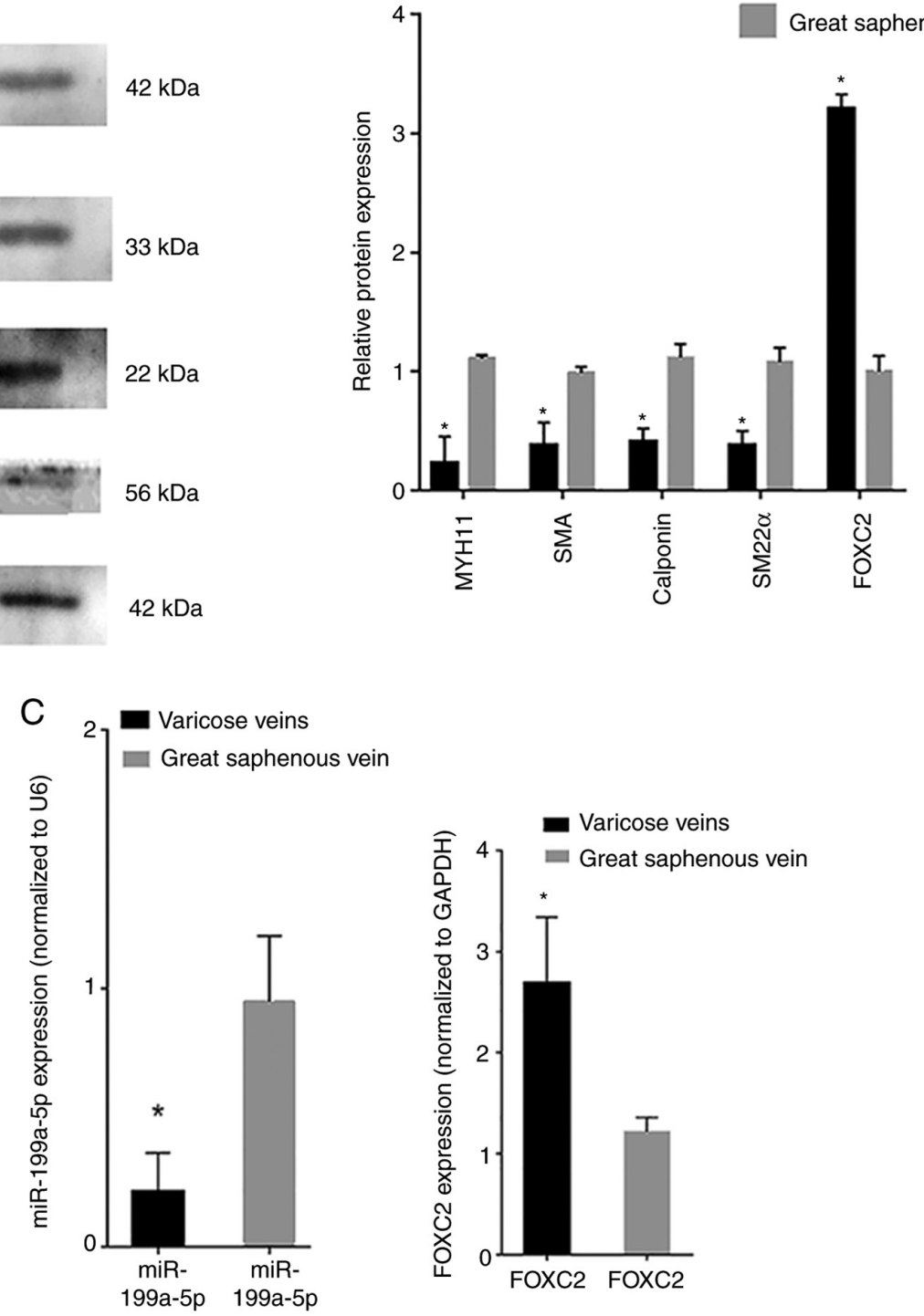

Figure 1. Expression levels of miR-199a-5p are decreased in human varicose vein tissues. (A) RT-qPCR revealed that the expression levels of VSMC differentiation biomarkers were decreased in varicose vein tissues $(n=10) .{ }^{*} \mathrm{P}<0.05$ vs. great saphenous vein tissues. (B) Western blot analysis revealed that the protein expression levels of VSMC differentiation biomarkers were decreased and the expression levels of FOXC2 were increased in varicose vein tissues $(\mathrm{n}=3) .{ }^{*} \mathrm{P}<0.05$ vs. great saphenous vein tissues. (C) miR-199a-5p was downregulated and FOXC2 was upregulated in varicose vein tissues, as determined by RT-qPCR. ( $\mathrm{n}=10)$. $\mathrm{P}<0.05$ vs. great saphenous vein tissues. miR-199a-5p, microRNA-199a-5p; FOXC2, forkhead box C2; MYH11, myosin heavy chain 11; RT-qPCR, Reverse transcription-quantitative PCR; SM22 $\alpha$, smooth muscle 22 $\alpha$; SMA, smooth muscle actin; VSMC, vascular smooth muscle cell. 

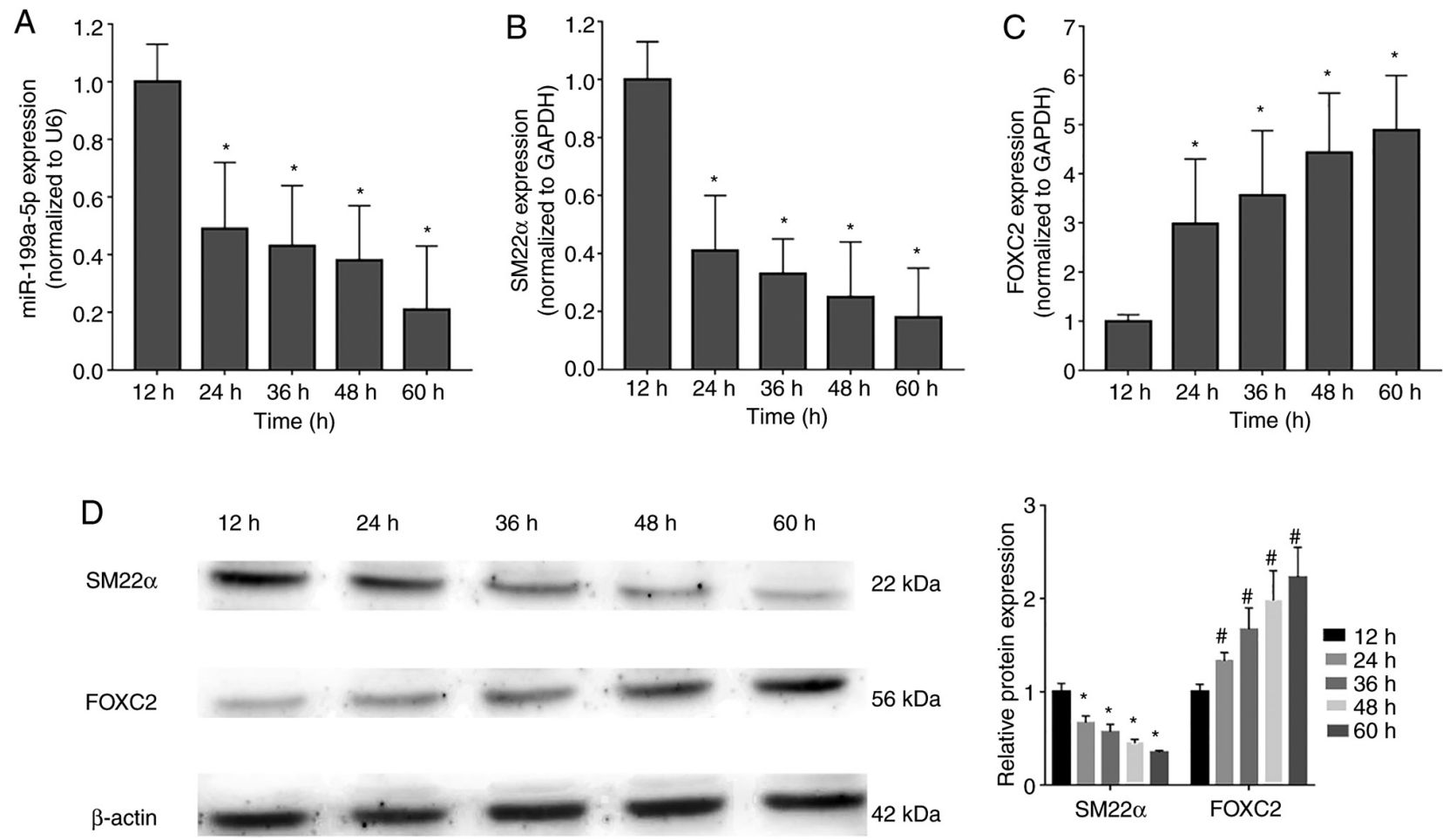

Figure 2. Time-dependent expression changes of miR-199a-5p, SM22 $\alpha$ and FOXC2 in proliferating VSMCs. Reverse transcription-quantitative PCR showed the time-dependent decrease in (A) miR-199a-5p and (B) SM22 $\alpha$ mRNA expression, and (C) a time-dependent increase in FOXC2 mRNA expression in VSMCs. "P<0.05 vs. 12-h group (n=3). (D) Western blot analysis revealed that SM22 $\alpha$ protein expression was downregulated, but FOXC2 was upregulated in VSMCs. ${ }^{*} \mathrm{P}<0.05$ vs. 12-h group ( $\left.\mathrm{n}=3\right) .{ }^{~} \mathrm{P}<0.05$ vs. 12-h group $(\mathrm{n}=3)$. FOXC2, forkhead box C2; miR-199a-5p, microRNA-199a-5p; SM22 $\alpha$, smooth muscle $22 \alpha$; VSMC, vascular smooth muscle cell.

transfection with miR-199a-5p mimics induced an increase in miR-199a-5p expression in VSMCs, whereas the miR-199a-5p inhibitor markedly reduced endogenous miR-199a-5p expression levels compared with the control (untransfected VSMCs) (Fig. 3A). In addition, VSMCs were transfected with miR-199a-5p mimics, NC mimics, miR-199a-5p inhibitor and $\mathrm{NC}$ inhibitor and FOXC2 protein expression was detected by western blotting (Fig. S1C). Western blotting showed that FOXC2 was upregulated in VSMCs transfected with inhibitor compared with NC inhibitor, FOXC2 was downregulated in VSMCs transfected with mimics compared with NC mimics. RT-qPCR and western blotting revealed that the mRNA and protein expression levels, respectively, of the phenotypic transition biomarkers SM22 $\alpha$, calponin, SMA and MYH11, which are involved in VSMC differentiation, were downregulated in cells transfected with the miR-199a-5p inhibitor (Fig. 3B and C), whereas miR-199a-5p overexpression was associated with upregulation of these phenotypic transition biomarkers. These results suggested that miR-199a-5p may regulate the expression of phenotypic transition biomarkers involved in VSMC differentiation.

miR-199a-5p regulates VSMC proliferation and migration. The effects of miR-199a-5p on VSMC migration were determined by performing Transwell migration and wound healing assays following miR-199a-5p mimics and inhibitor transfection. The results revealed that miR-199a-5p knockdown significantly promoted the migratory ability of VSMCs, whereas transfection with miR-199a-5p mimics significantly inhibited the migratory ability of VSMCs
(Fig. 4A). A marked increase in cell migration was also detected by wound healing assay after transfection with the miR-199a-5p inhibitor at $48 \mathrm{~h}$, whereas cells transfected with the miR-199a-5p mimics exhibited reduced cell migration ability at $48 \mathrm{~h}$ (Fig. 4B).

A CCK-8 proliferation assay was performed to detect the proliferative ability of VSMCs. VSMCs were transfection with the miR-199a-5p mimics or inhibitor, and cell proliferation was measured at 24, 48 and $72 \mathrm{~h}$. Untransfected cells were considered the control group. VSMCs overexpressing miR-199a-5p exhibited a decrease in proliferation compared with that in the control cells (Fig. 4C), whereas VSMCs with miR-199a-5p knockdown exhibited increased proliferation. Western blot analysis revealed that the proliferation biomarker PCNA was downregulated in miR-199a-5p mimics-transfected cells, but upregulated in miR-199a-5p inhibitor-transfected cells (Fig. 4D). These results indicated that miR-199a-5p may decrease VSMC proliferation and migration.

FOXC2 is a target gene of miR-199a-5p. Bioinformatics analysis revealed putative miR-199a-5p target sites in the FOXC2 3'-UTR region (Fig. 5). A dual luciferase reporter assay was performed to confirm the effects of miR-199a-5p on FOXC2. 293T cells transfected with miR-199a-5p mimics reduced luciferase activity in cells expressing FOXC2-WT compared with that in untransfected control cells or 293T cells transfected with NC mimics and FOXC2-MUT/FOXC2-WT (Fig. 5).

The pcDNA3.1-FOXC2 overexpression vector and si-FOXC2 were transfected into VSMCs for rescue experiments. 

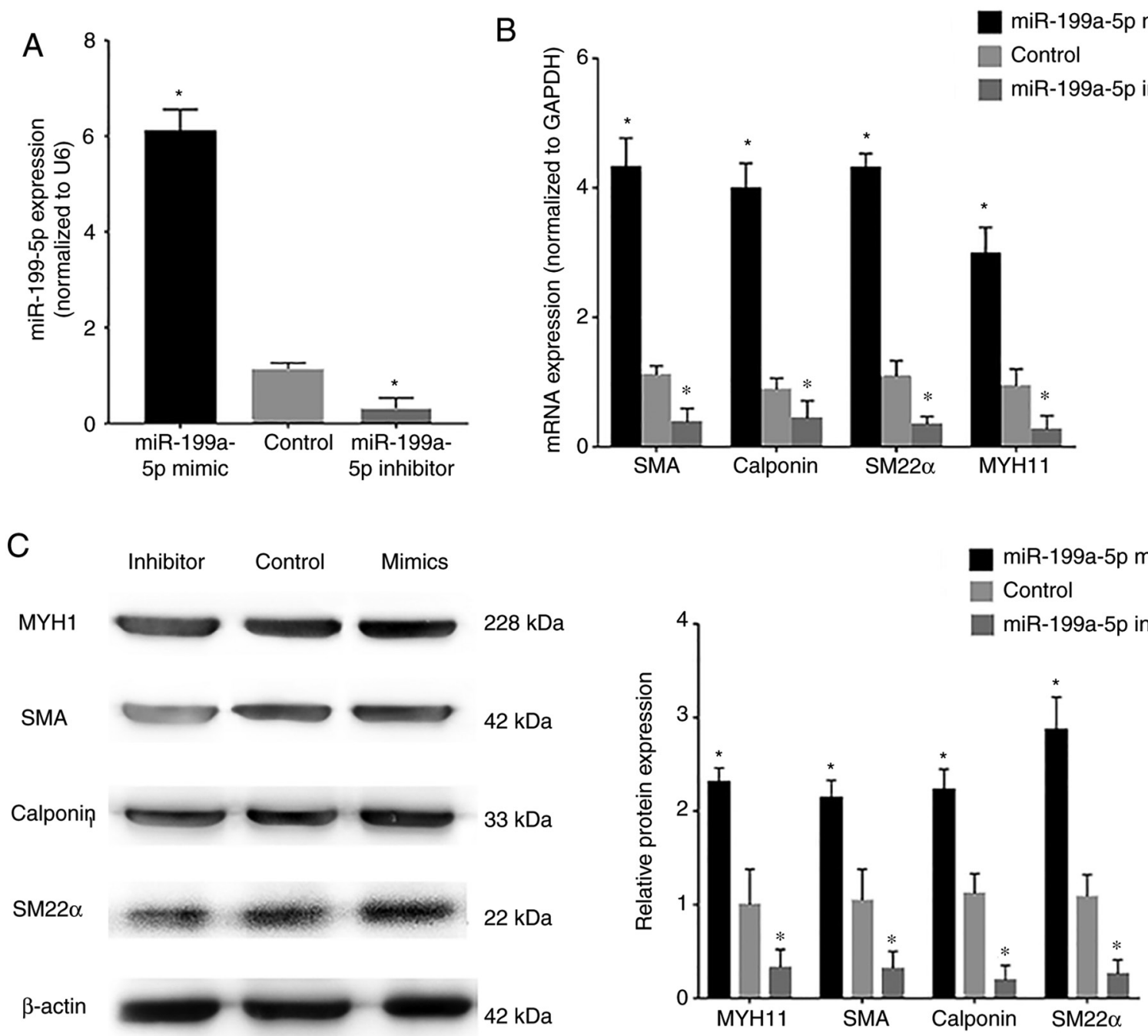

Figure 3. miR-199a-5p regulates the expression levels of VSMC biomarkers. (A) Reverse transcription-quantitative PCR confirmed that miR-199a-5p was overexpressed and knocked down post-transfection with the miR-199a-5p mimics and inhibitor, respectively $(\mathrm{n}=3)$. (B) Overexpression or knockdown of miR-199a-5p promoted or inhibited the expression of VSMC differentiation biomarkers, respectively. ${ }^{*} \mathrm{P}<0.05$ vs. control ( $\left.\mathrm{n}=10\right)$. (C) Western blot analysis of VSMC differentiation biomarkers. "P<0.05 vs. control (n=3). miR-199a-5p, microRNA-199a-5p; MYH11, myosin heavy chain 11; SM22 $\alpha$, smooth muscle 22 $\alpha$; SMA, smooth muscle actin; VSMC, vascular smooth muscle cell.

VSMCs were transfected with FOXC2 vector, pCDNA3.1 $\mathrm{NC}$, si-FOXC2 and siNC, and the transfection efficiency was confirmed by western blotting (Fig. S2). si-FOXC2 decreased the expression levels of FOXC2 compared with siNC, which increased the expression levels of MYH11, SMA, Calponin and SM22. FOXC2 vector increased the expression levels of FOXC2 compared with pcDNA3.1 NC, which decreased the expression levels of MYH11, SMA, Calponin and SM22. RT-qPCR was also performed to verify the transfection efficiency of the pcDNA3.1-FOXC2 vector and FOXC2 siRNA; untransfected cells were considered the control group. pcDNA3.1-FOXC2 vector transfection increased the expression levels of FOXC2 in VSMCs compared with those in the control group (Fig. 6A). VSMCs transfected with miR-199a-5p mimics + pcDNA3.1-FOXC2 vector exhibited higher proliferation (Fig. 6B) and migration (Fig. 6C) compared with that in cells transfected with only the miR-199a-5p mimics. Phenotypic transition biomarkers involved in VSMC differentiation, such as SM22 $\alpha$, calponin, SMA and MYH11, were downregulated in VSMCs transfected with miR-199a-5p mimics + pcDNA3.1-FOXC2 vector compared with those in VSMCs transfected with the miR-199a-5p mimics only (Fig. 6D and E).

In addition, the role of FOXC2 in VSMC proliferation, migration and phenotypic switch was assessed. Expression levels of the differentiation biomarkers were increased in VSMCs transfected with the FOXC2 siRNA compared with those in the control group (Fig. 6F). In addition, a marked decrease in cell proliferation was detected in cells transfected with the FOXC2 siRNA (Fig. 6G). As shown in Fig. 6H, a marked decrease in the migration of cells transfected with the FOXC2 siRNA was revealed at $48 \mathrm{~h}$.

\section{Discussion}

Major biological functions of the vascular system are performed in VSMCs. Notably, phenotypic switching of VSMCs has been reported to serve a critical role in varicose vein pathogenesis (4). VSMCs acquire proliferative and migratory abilities when they transition to a synthetic phenotype, resulting in 

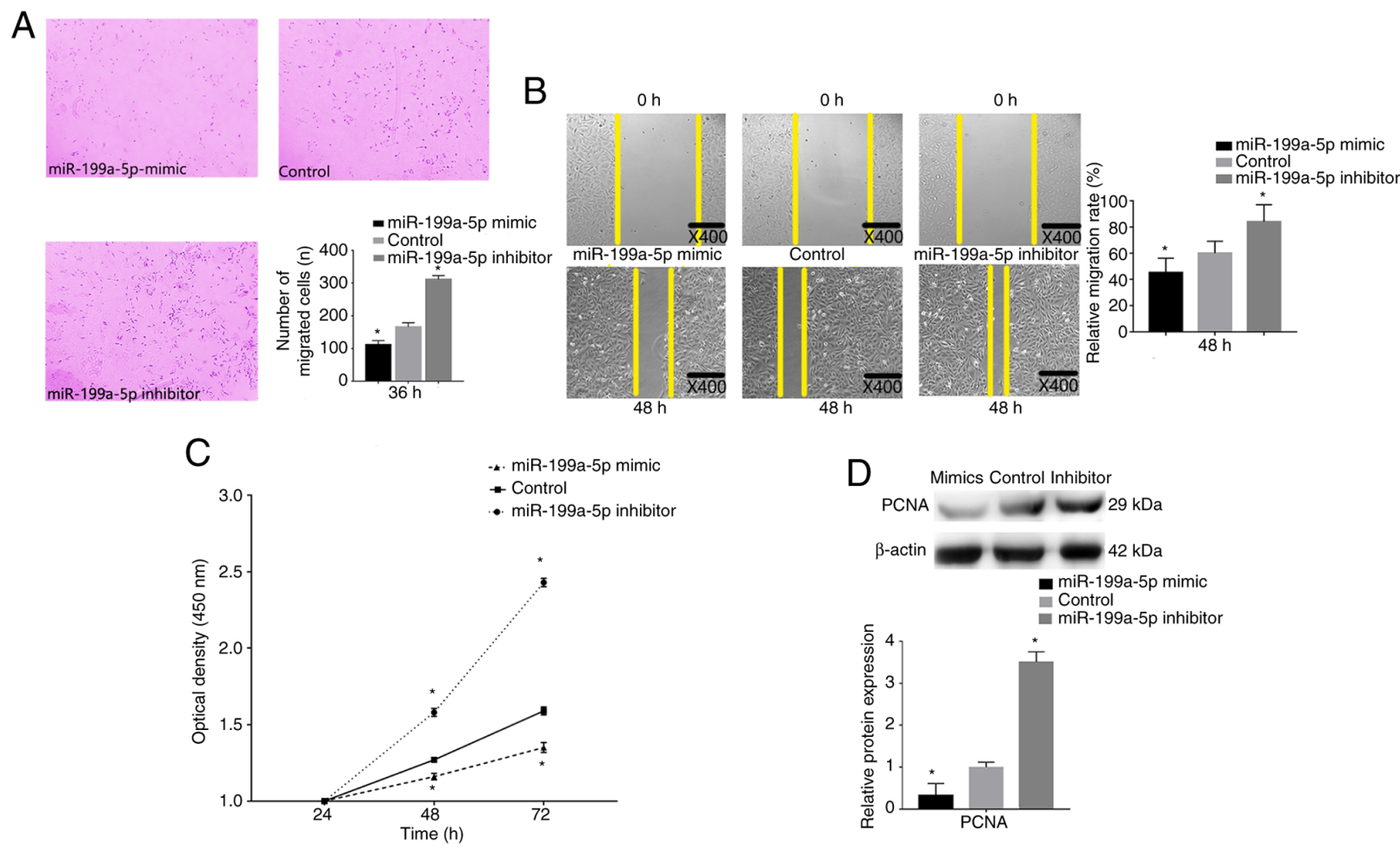

Figure 4. High expression of miR-199a-5p inhibits proliferation and migration. (A) Transwell migration assays revealed that knockdown of miR-199a-5p promoted VSMC migration compared with untransfected VSMCs, whereas VSMC transfected with miR-199a-5p mimics inhibited VSMC migration. Magnification, $x 40 .{ }^{*} \mathrm{P}<0.05$ vs. control (n=3). (B) Wound healing assay revealed the enhanced migration of VSMCs transfected with the miR-199a-5p inhibitor. (C) Cell Counting Kit-8 revealed that VSMC proliferation was enhanced after transfection with the miR-199a-5p inhibitor. ${ }^{*} \mathrm{P}<0.05$ vs. control $(\mathrm{n}=10)$. (D) Western blotting revealed that PCNA was upregulated in VSMCs transfected with the miR-199a-5p inhibitor. ${ }^{*} \mathrm{P}<0.05$ vs. control ( $=3$ ). miR-199a-5p, microRNA-199a-5p; PCNA, proliferating cell nuclear antigen; VSMC, vascular smooth muscle cell.

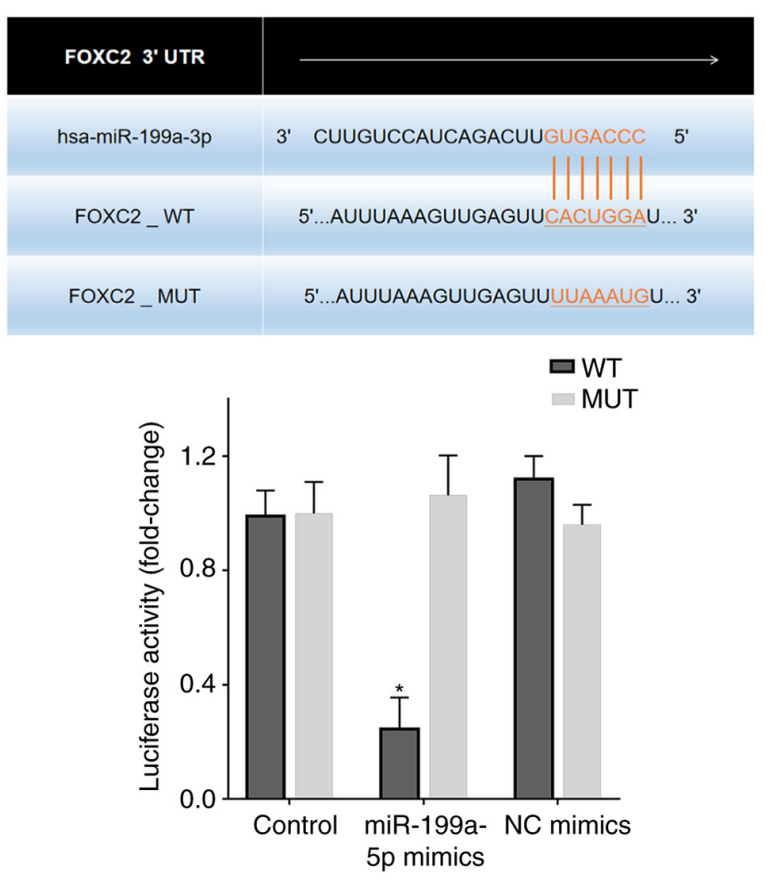

Figure 5. Association between miR-199a-5p and FOXC2. Putative miR-199a-5p target sites were identified in the 3'-UTR of FOXC2. Dual luciferase reporter assay revealed that miR-199a-5p reduced the luciferase activity of 293T cells transfected with the WT 3'-UTR of FOXC2 compared with that in untransfected cells or 293T cells transfected with NC mimic and FOXC2-MUT/FOXC2-WT. ${ }^{*} \mathrm{P}<0.05$ vs. Control or NC mimics or miR-199a-5p mimics/FOXC2-MUT ( $n=10)$. 3'-UTR, 3'-untranslated region; FOXC2, forkhead box C2; miR-199a-5p, microRNA-199a-5p; MUT, mutant; WT, wild-type; NC, negative control. vascular remodeling. In the present study, contractile markers, such as SM22 $\alpha$, SMA, calponin and MYH11, were downregulated in varicose vein tissues compared with those in normal vein tissues, suggesting that the contractile phenotype is a normal phenotype of VSMCs. To confirm that miR-199a-5p specifically participated in regulating VSMC proliferation, the expression levels of miR-199a-5p were detected in VSMCs at different time points, which revealed that miR-199a-5p was downregulated in VSMCs during cell proliferation.

In the present study, RT-qPCR confirmed that the expression of miR-199a-5p was downregulated in varicose vein tissues, which revealed that miR-199a-5p may promote VSMC transition to the synthetic phenotype by reducing VSMC contractile markers, such as SM22 $\alpha$, SMA, calponin and MYH11. The function of miR-199a-5p has been reported in several other diseases. For example, miR-199a-5p downregulation has been reported to promote non-small cell lung cancer proliferation (18). In addition, miR-199a-5p upregulation could inhibit the progression of papillary thyroid carcinoma by reducing the migratory ability of cells (19). In addition, miR-199a-5p upregulation promoted colorectal cancer cell proliferation and migration (20). Therefore, the roles of miR-199a-5p vary depending on the type of tissues involved in the disease. Ahmadi et al (18) showed that the downregulation of miR-199a-5p promoted lung cancer cell proliferation and migration via targeting GRP78 within the unfolded protein response pathway. Ma et al (19) reported that the downregulation of miR-199a-5p promoted papillary thyroid carcinoma cell proliferation and migration by targeting SNAI1. Thus, the 
A

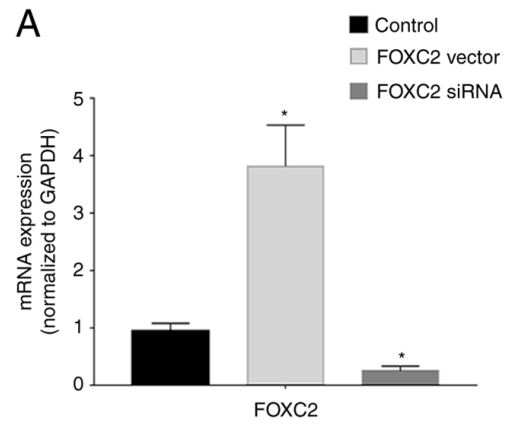

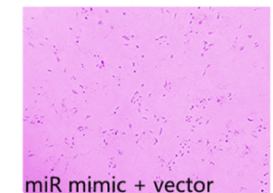

miR mimic + vector

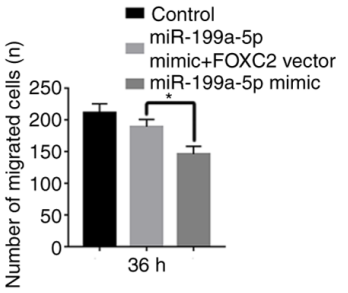

E
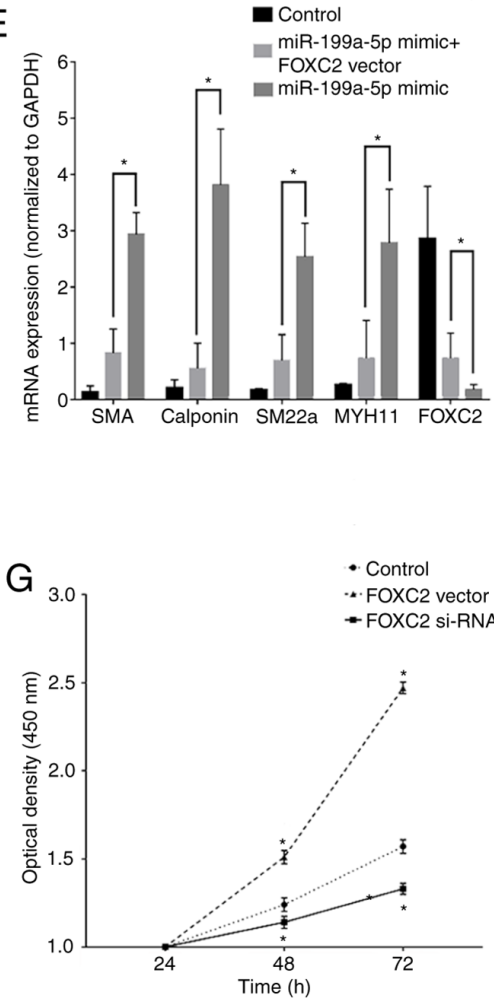

D
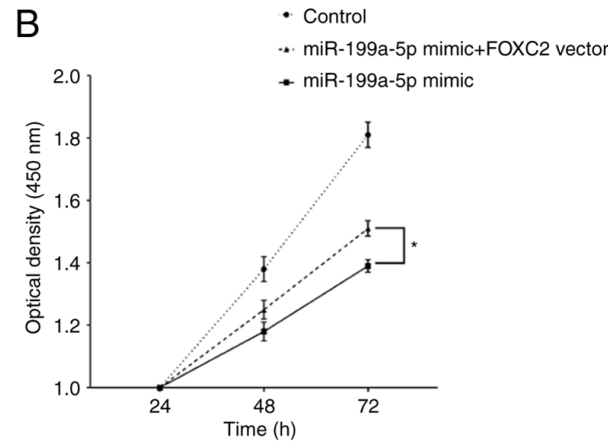
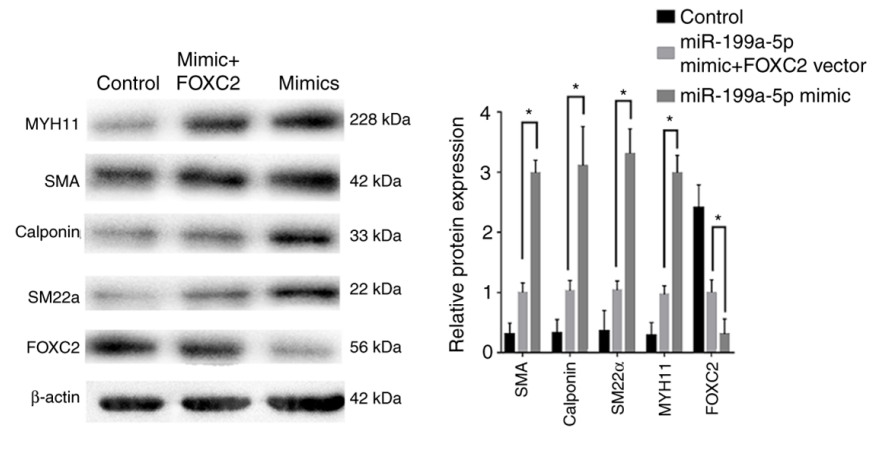

$\mathrm{F}$

- Control

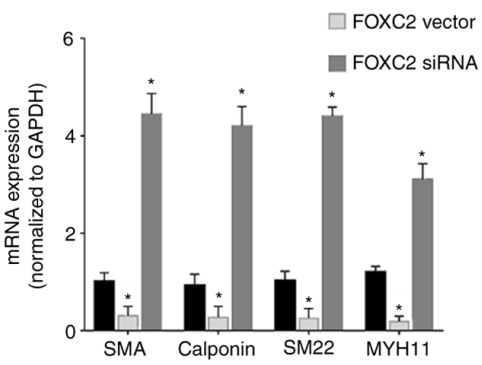

$\mathrm{H}$
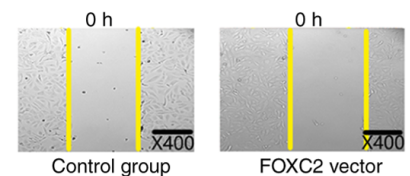

$\mathrm{Oh}$
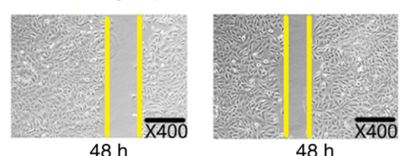

FOXC2 SiRNA

$48 \mathrm{~h}$
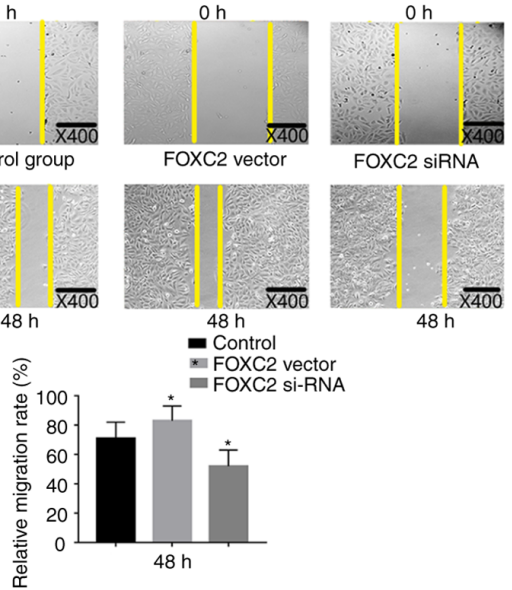

$48 \mathrm{~h}$

Figure 6. FOXC2 rescue experiments. (A) RT-qPCR confirmed that FOXC2 was overexpressed and knocked down post-transfection with pcDNA3.1-FOXC2 vector or FOXC2 siRNA, respectively. " $\mathrm{P}<0.05$ vs. control ( $\mathrm{n}=3$ ). (B) CCK-8 confirmed that VSMC proliferation was enhanced after transfection with miR-199a-5p mimics + FOXC2 vector compared with miR-199a-5p mimics alone. ${ }^{*} \mathrm{P}<0.05(\mathrm{n}=10)$. (C) Transwell migration assays revealed that FOXC2 enhanced VSMC migration. Magnification, $\mathrm{x} 40 .{ }^{*} \mathrm{P}<0.05(\mathrm{n}=10)$. (D) Western blot analysis revealed that the expression levels of VSMC differentiation biomarkers were decreased in cells transfected with miR-199a-5p mimics + FOXC2 vector compared with those in cells transfected with miR-199a-5p mimics only. "P<0.05 (n=3). (E) RT-qPCR revealed that FOXC3 decreased the expression levels of VSMC differentiation biomarkers compared with those in cells transfected with miR-199a-5p mimics only. ${ }^{*} \mathrm{P}<0.05$ (n=10). (F) RT-qPCR was used to detect the expression levels of phenotypic transition biomarkers. ${ }^{*} \mathrm{P}<0.05$ vs. control ( $\mathrm{n}=10)$. (G) CCK-8 confirmed that proliferation of VSMCs was reduced in response to FOXC2 silencing, but increased in response to FOXC2 overexpression. " $\mathrm{P}<0.05$ vs. control $(\mathrm{n}=3)$. (H) Wound healing assay revealed that migration of VSMCs was reduced post-transfection with the FOXC2 siRNA, but increased following the overexpression of FOXC2 compared with the control group. " $\mathrm{P}<0.05$ vs. control ( $\mathrm{n}=3$ ). FOXC2, forkhead box C2; miR-199a-5p, microRNA-199a-5p; MYH11, myosin heavy chain 11; RT-qPCR, reverse transcription-quantitative PCR; siRNA, small interfering RNA; SM22 $\alpha$, smooth muscle 22 $\alpha$; SMA, smooth muscle actin; VSMC, vascular smooth muscle cell; CCK-8, Cell Counting Kit-8. 
present findings are consistent with the findings of previous studies $(18,19)$, indicating that miR-199a-5p downregulation may promote VSMC proliferation and migration. In the present study, VSMCs were transfected with a miR-199a-5p inhibitor, after which, the expression levels of the contractile markers SM22 $\alpha$, SMA, calponin and MYH11 were downregulated, suggesting that VSMCs switched to a synthetic phenotype. Conversely, it has previously been reported that miR-199a-5p upregulation may promote cell proliferation and metastasis in cervical carcinoma (21), and miR-199a-5p downregulation could inhibit osteoblast differentiation (5). Therefore, the function of miR-199a-5p may vary in different cell types.

Phenotypic switching of VSMCs serves as an initial stage in varicose vein formation; however, the mechanism involved in phenotypic switching during varicose vein formation remains unclear. The role of miR-199a-5p also depends on its target genes. After confirming that miR-199a-5p was downregulated in varicose vein tissues, the present study aimed to identify the potential target genes, as miRNAs regulate translation by binding to the 3'-UTRs of target genes. FOXC2 is a pathogenic gene that has been reported to be associated with venous valve dysfunction (22). A number of studies have found that FOXC2 plays an important role in a number of cancers. FOXC2 has been reported to be overexpressed in breast (23), stomach (24), cervical (25) and ovarian (26) cancer. In addition, the transcription factor FOXC2 has been shown to promote cell proliferation and also induce the EMT via multiple pathways in malignancies. Moreover, FOXC2 can also act as a crucial mediator in both angiogenesis and lymphangiogenesis (27), thus it could act as a potential transcriptional mediator involved in increased pathological angiogenesis and neovascularization (28). The present study confirmed that FOXC2 was upregulated in varicose vein tissues and promoted VSMC proliferation; this finding is consistent with that of a previous report (29). After identifying an opposite trend in miR-199a-5p and FOXC2 expression levels in varicose vein tissues, it was hypothesized that miR-199a-5p downregulation seemed to be associated with varicose vein pathogenesis by binding FOXC2 These findings indicated that miR-199a-5p downregulation may promote the development of varicose veins by upregulating FOXC2.

In conclusion, miR-199a-5p was downregulated in varicose vein tissues compared with that in normal vein tissues. In addition, miR-199a-5p inhibited FOXC2 by targeting the 3'-UTR, which regulated VSMC phenotypic switching. Therefore, the miR-199a-5p/FOXC2 axis may provide a novel mechanistic insight into the pathogenesis of varicose veins and may serve as a promising diagnostic biomarker and therapeutic target for the treatment of varicose veins.

\section{Acknowledgements}

Not applicable.

\section{Funding}

This work was partly supported by the Development of Science and Technology in Jilin Province Project, China (grant no. 20190303045SF).

\section{Availability of data and materials}

The datasets used and/or analyzed during the current study are available from the corresponding author on reasonable request.

\section{Authors' contributions}

YC and ZC designed and supervised the study. YC and WW performed the experiments. WW, XJ and LL analyzed and interpreted the data. YC and ZC confirm the authenticity of all the raw data. All authors have read and approved the final manuscript.

\section{Ethics approval and consent to participate}

The present study was conducted in accordance with the Declaration of Helsinki and was approved by the Ethics Committee of Qianwei Hospital of Jilin Province (approval no. QW202000224; Changchun, China). All patients agreed to the use of their samples in scientific research and provided written informed consent.

\section{Patient consent for publication}

Not applicable.

\section{Competing interests}

The authors declare that they have no competing interests.

\section{References}

1. Piazza G. Varicose veins. Circulation 130: 582-587, 2014.

2. Jacobs BN, Andraska EA, Obi AT and Wakefield TW: Pathophysiology of varicose veins. J Vasc Surg Venous Lymphat Disord 5: 460-467, 2017.

3. Xu Y, Bei Y, Li Y and Chu H: Phenotypic and functional transformation in smooth muscle cells derived from varicose veins. J Vasc Surg Venous Lymphat Disord 5: 723-733, 2017.

4. Huang X, Liu Z, Shen L, Jin Y, Xu G, Zhang Z, Fang C, Guan W and Liu C: Augmentation of miR-202 in varicose veins modulates phenotypic transition of vascular smooth muscle cells by targeting proliferator-activated receptor- $\gamma$ coactivator-1 $\alpha$. J Cell Biochem 120: 10031-10042, 2019.

5. Qi XB, Jia B, Wang W, Xu GH, Guo JC, Li X and Liu JN: Role of miR-199a-5p in osteoblast differentiation by targeting TET2. Gene 726: 144193, 2020.

6. Bao N, Fang B, Lv H, Jiang Y, Chen F, Wang Z and Ma H: Upregulation of miR-199a-5p protects spinal cord against ischemia/reperfusion-induced injury via downregulation of ECE1 in rat. Cell Mol Neurobiol 38: 1293-1303, 2018.

7. Zhu H: Forkhead box transcription factors in embryonic heart development and congenital heart disease. Life Sci 144: 194-201, 2016.

8. Liu Y, Liu G, Zhang H and Wang J: MiRNA-199a-5p influences pulmonary artery hypertension via downregulating Smad3. Biochem Biophys Res Commun 473: 859-866, 2016.

9. Huang Y, Zhang S, Fang X, Qin L, Fan Y, Ding D, Liu X and Xie M: Plasma miR-199a-5p is increased in neutrophilic phenotype asthma patients and negatively correlated with pulmonary function. PLoS One 13: e0193502, 2018.

10. Agarwal V, Bell GW, Nam JW and Bartel DP: Predicting effective microRNA target sites in mammalian mRNAs. Elife 4: $\mathrm{e} 05005,2015$.

11. Mellor RH, Brice G, Stanton AW, French J, Smith A, Jeffery S, Levick JR, Burnand KG and Mortimer PS; Lymphoedema Research Consortium: Mutations in FOXC2 are strongly associated with primary valve failure in veins of the lower limb. Circulation 115: 1912-1920, 2007. 
12. Guo Z, Luo C, Zhu T, Li L and Zhang W: Elevated c-fos expression is correlated with phenotypic switching of human vascular smooth muscle cells derived from lower limb venous varicosities. J Vasc Surg Venous Lymphat Disord 9: 242-251, 2021.

13. Surendran S, Ramegowda KS, Suresh A, Binil Raj SS Lakkappa RK, Kamalapurkar G, Radhakrishnan $\mathrm{N}$ and C Kartha C: Arterialization and anomalous vein wall remodeling in varicose veins is associated with upregulated FoxC2-Dll4 pathway. Lab Invest 96: 399-408, 2016.

14. Baeten JT and Lilly B: Notch signaling in vascular smooth muscle cells. Adv Pharmacol 78: 351-382, 2017.

15. Porter JM and Moneta GL: Reporting standards in venous disease: An update. International Consensus Committee on Chronic Venous Disease. J Vasc Surg 21: 635-645, 1995.

16. Livak KJ and Schmittgen TD: Analysis of relative gene expression data using real-time quantitative PCR and the 2(-Delta Delta C(T)) method. Methods 25: 402-408, 2001

17. Tang Y, Yu S, Liu Y, Zhang J, Han L and Xu Z: MicroRNA-124 controls human vascular smooth muscle cell phenotypic switch via Sp1. Am J Physiol Heart Circ Physiol 313: H641-H649, 2017.

18. Ahmadi A, Khansarinejad B, Hosseinkhani S, Ghanei M and Mowla SJ: miR-199a-5p and miR-495 target GRP78 within UPR pathway of lung cancer. Gene 620: 15-22, 2017.

19. Ma S, Jia W and Ni S: miR-199a-5p inhibits the progression of papillary thyroid carcinoma by targeting SNAI1. Biochem Biophys Res Commun 497: 181-186, 2018.

20. Zhu QD, Zhou QQ, Dong L, Huang Z, Wu F and Deng X: MiR-199a-5p inhibits the growth and metastasis of colorectal cancer cells by targeting ROCK1. Technol Cancer Res Treat 17: 1533034618775509,2018

21. Qu D, Yang Y and Huang X: miR-199a-5p promotes proliferation and metastasis and epithelial-mesenchymal transition through targeting PIAS3 in cervical carcinoma. J Cell Biochem 120: 13562-13572, 2019.
22. Lim CS and Davies AH: Pathogenesis of primary varicose veins Br J Surg 96: 1231-1242, 2009.

23. Pham TND, Perez White BE,Zhao H, Mortazavi Fand TonettiDA Protein kinase $\mathrm{C} \alpha$ enhances migration of breast cancer cells through FOXC2-mediated repression of p120-catenin. BMC Cancer 17: 832, 2017.

24. Ren YH, Liu KJ, Wang M, Yu YN, Yang K, Chen Q, Yu B, Wang W, Li QW, Wang J, et al: De-SUMOylation of FOXC2 by SENP3 promotes the epithelial-mesenchymal transition in gastric cancer cells. Oncotarget 5: 7093-7104, 2014.

25. Zheng CH, Quan Y, Li YY, Deng WG, Shao WJ and Fu Y: Expression of transcription factor FOXC2 in cervical cancer and effects of silencing on cervical cancer cell proliferation. Asian Pac J Cancer Prev 15: 1589-1595, 2014.

26. Liu B, Han SM, Tang XY, Han L and Li CZ: Overexpressed FOXC2 in ovarian cancer enhances the epithelial-to-mesenchymal transition and invasion of ovarian cancer cells. Oncol Rep 31: 2545-2554, 2014.

27. Wu X and Liu NF: FOXC2 transcription factor: A novel regulator of lymphangiogenesis. Lymphology 44: 35-41, 2011.

28. Sano H, Leboeuf JP, Novitskiy SV, Seo S, Zaja-Milatovic S, Dikov MM and Kume T: The Foxc2 transcription factor regulates tumor angiogenesis. Biochem Biophys Res Commun 392: 201-206, 2010

29. Zhang C, Li H and Guo X: FOXC2-AS1 regulates phenotypic transition, proliferation and migration of human great saphenous vein smooth muscle cells. Biol Res 52: 59, 2019.

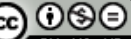

This work is licensed under a Creative Commons Attribution-NonCommercial-NoDerivatives 4.0 International (CC BY-NC-ND 4.0) License. 